\title{
Tele-Health: Integrated Remote Disease Diagnosis and Treatment Platform
}

\author{
Pravin Wararkar, Shashikant Patil, Sunil Chaudhari \\ Department of Electronics \& Telecommunication Engineering, \\ SVKMs NMIMS (Deemed-to-be UNIVERSITY), MPSTME, MPTP Shirpur Campus, Shirpur \\ *Corresponding Author: Pravin Wararkar, Department of Electronics \& Telecommunication \\ Engineering,

\begin{abstract}
The new era of telecommunication networks and updating of new technologies including internet can be used in the support of the tele-medicine field. Tele-medicine or tele-health is nothing but interaction between a doctor and a patient from remote places. Due to development of such technologies many remote areas have maintained a good health care system. This is required in places such as Africa where there is no convenient transport system to travel upto the hospital. Therefore in this research work, we have discussed the application, implementation and problems of the telemedicine in day to day life. This technology has been used in many places but is still rare in countries like India.
\end{abstract}

Keywords: Tele-medicine, Tele-health

\section{INTRODUCTION}

With emerging diversification of technologies in the new era, field of medicine and medical health are also on the route of standardization, prevention of high quality and level, increasing efficiency economic and convenient cure of evolving diseases. E-health has created exceptional opportunities and success. Use of audio video device, smart phone app, and internet create a platform to monitor disease and instruct health management. Telemedicine is helpful for carry out of operation such as education, rehabilitation guidance that provide patient with long term guidance, health risk and drug assessment. Technologies such as IOT (Internet of Things) \& RFID (Radio Frequency Identification) have evolved in medical field for diagnosis of chronic diseases such as Alzheimer's, diabetes, heart abnormalities, high blood pressure, asthma, osteoarthritis. Tele-health system helps in diagnosis of elderly individuals over long distances, it capture and store vital signs and clinical data and forward for further analysis to caregivers, provide them with private healthcare management, conformity that improves the quality of personal healthcare and efficient medical and social assistance. Wearable health monitoring technologies are paramount with provision of early health diagnosis and detection of signs, notify caregivers in critical situation, find correlation between health and lifestyle, uplift of healthcare in remote areas thus helping health providers to initiate patient oriented processes with assessment of real time data. Tele-health together is a mixture of medicine, technology and internet that come all together to run health data repository giving internet access and collection of patient oriented medical information for later evaluation thus offering various set of experimental solutions for different Tele-medical application, home monitoring and Tele-consultation to its innumerous clients. Relevant data related to client's health can be entered whenever and wherever they may arise. The software platform and report generating system provide dedicated applications that interact with support staff and Tele-assisted and gives the platform of Tele-health the benefit of graphical and statistical evaluation of data generated.

\section{Application OF TELEMEDicine}

\subsection{Tele-echocardiography}

Tele-echocardiography is the method by which we can study the heart regarding its study or to find the problem of heart. It is a technology by which we can study the heart anywhere anytime with the 
help of the machine which will find the problem by waves transmission through the body. The similar type of machine is present at the receiver side. With this technology a person can be treated anytime. The problems are that a specialist is required to handle the machine also it is way too costly so it has to be handled with care. Also it is difficult to implement this because it is still not clear that the treatment is covered in medical insurance or not. The technology has its advantages and disadvantages. This technology has also been used on the astronaut for space studies to know the functioning of the heart in outer space so that they can use this data for further studies and international space projects such as the mission to mars etc.

\subsection{Tele-Nursing}

Today the use of tele-nursing is used in which the customer is kept under supervision when they are released from the hospital. It is providing therapeutic services by giving education, follow up, proper nursing and care by communicating with them on daily bases using telecommunication technology such as webcams, computers etc. in this service the family and patient are thought to use the machines and the manuals are given to use for daily check-up and then the output data is given to the hospital where the keep the check on the improvement of the patient. This has been a success in treatment of issues like backache, asthma, etc.

The tele-health diagnosis also has limitation such as in case of regular treatments or the initial stage of the disease it may not be possible to correctly determine it. So in this case proper supervision is required. It is seen that about $20 \%$ of the disease need expert supervision rest can be diagnosed by online web based applications. Proper high speed internet is necessary for efficient working at both the ends.

Expert's help is needed in the following situations:

- When patients problems cannot be answered perfectly by the system.

- When the person is fully not convinced by the results showing on the application.

- When processing time is too short resulting in really high probability of incorrect diagnosis.

- It is also sometimes possible that the doctors are not available for the patient for direct video communication and service so there are two types of workings that are use namely

\section{SYNCHRONOUS AND ASYNCHRONOUS TYPE OF SERVICES}

\subsection{Synchronous Diagnosis Subsystem}

In this type the patients are addressed with the webcam video conference and the result of the diagnosis is provided to the doctor at that instant only and the doctor asks the patient about the improvement and problem himself.

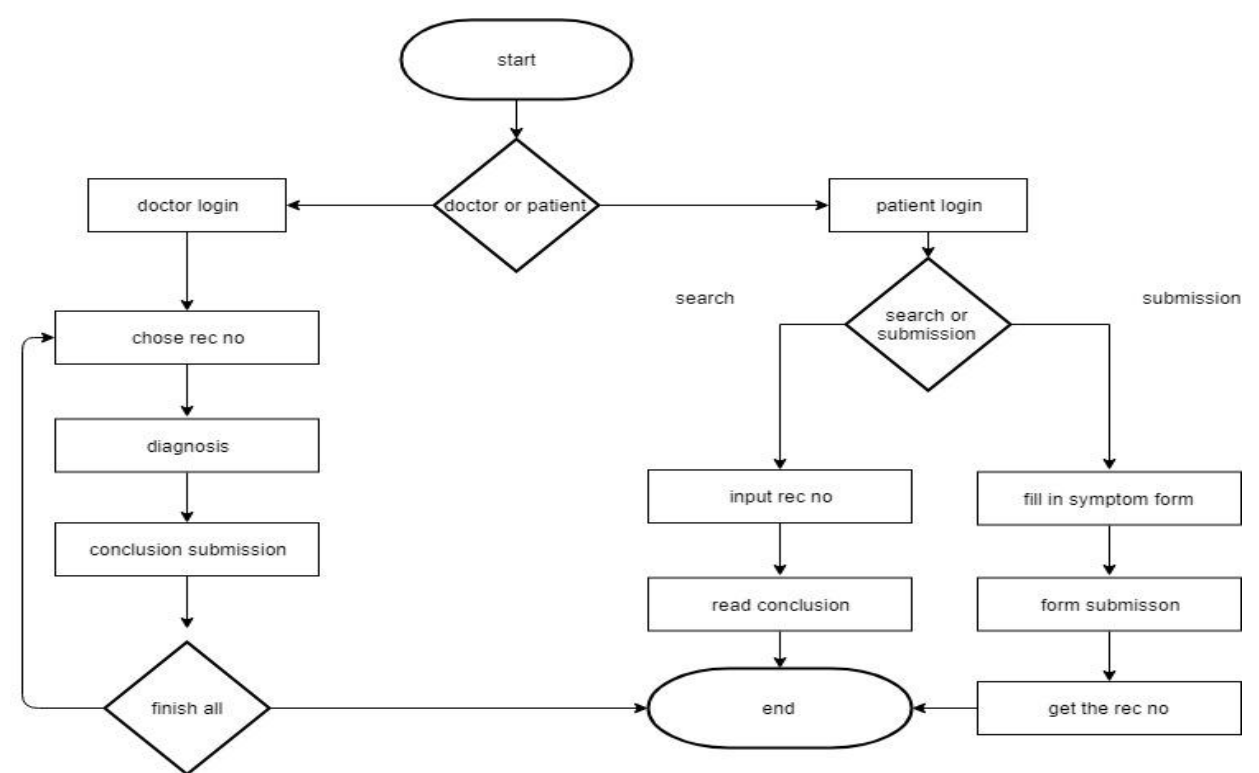

Figure1. Synchronous diagnosis subsystem 


\subsection{Tele-Health Using Mobile Communication}

The concept of tele-health also can be implied in mobiles as of today various wearable sensors are available which keep the track of the persons heart rate, the number of calories burnt etc. the bands transfer the data to the

Mobile. Also the high resolution videos stream using the Smartphone's internal CCD video camera can be done. It is also an effective way tokeep track of our health on daily bases.

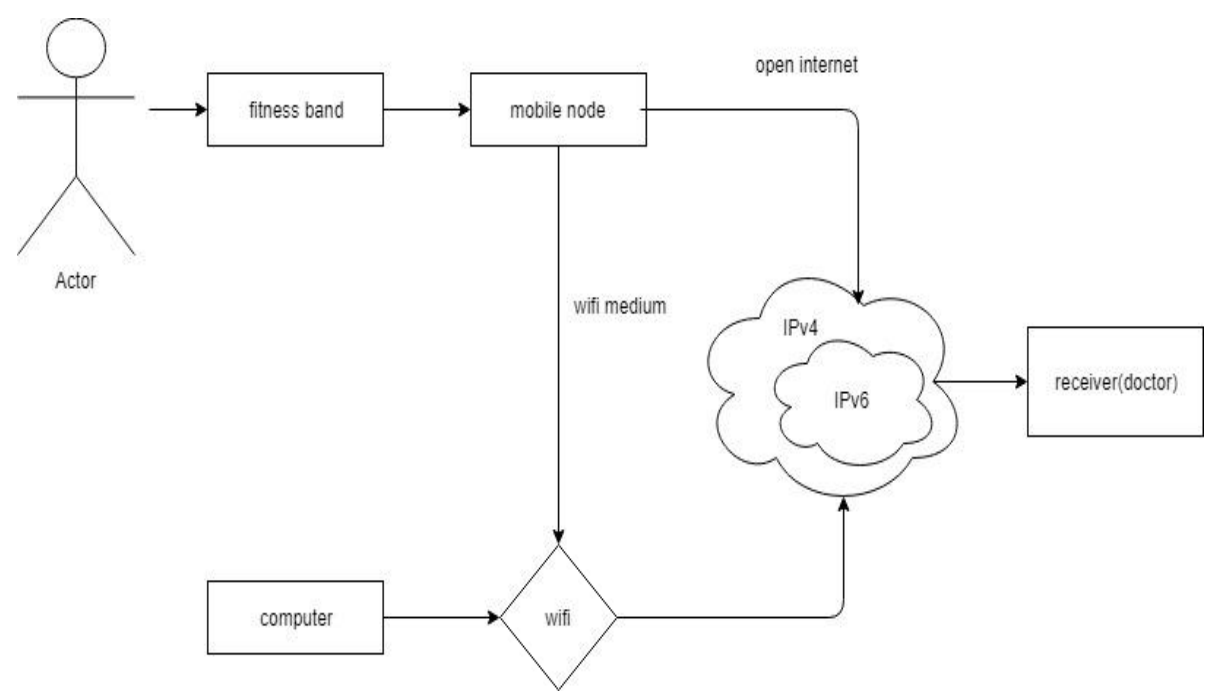

Figure2. Tele-health using mobile communication

\section{IMPLEMENTATION OF TELE-HEALTHCARE Systems}

\subsection{Affective Interfaces Fortele-hhc}

Along with the physical checkup it is equally important to have an emotional monitoring too. Many a times emotional disturbance can be the root cause of a major disease, hence emotional check up is also important. Different technologies have been used in order to gather the emotional signals of the user(patient).

The basic idea here is to synchronize the emotional signals with physical check up in order to get the effective information about the health of the patient. System architecture is being made for the monitoring of the emotional of the user.

The following block diagram explains the working of how to read the emotional disturbance of the user:

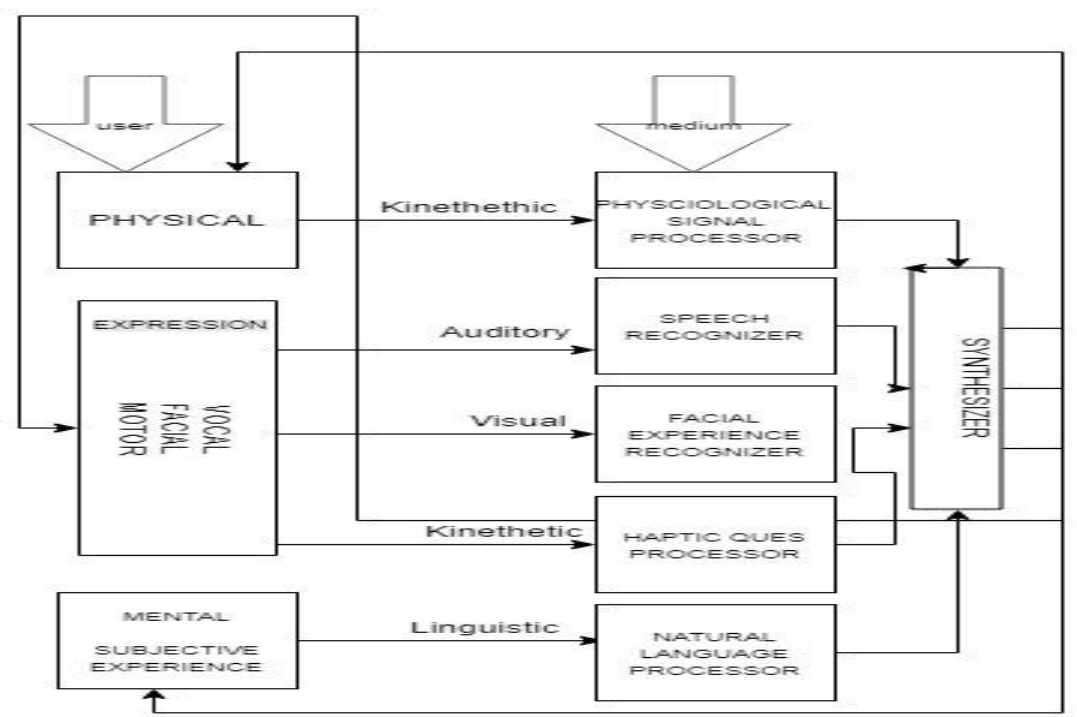

Figure3. How to read the emotional disturbance of the use 
In this model system first the patient is observed via multi sensors and then the output of the sensors is sent to the clinician who they recognizes the report or output and prescribe accordingly.

Main objectives of this model are:

- Database for each type of emotion

- Identifying similar emotions and generating their statistical data

- Inferring emotions and offering emotions about the patients next state.

Providing the feedback accordingly. Currently people are working in to detect and monitor patients ongoing emotional state via multimedia

Research is still going on this concept as to how one can suggest the patient to move from one integrating the model that shows the emotional state of the patient with expert's system willhelp to assess the patient's health collectively.

\section{REMOTE CONTROL SONOGRAPHY}

The aim here is to develop a low cost remote sonography system for tele-expertise in real time.

- Here we try to visualise the sonography images that has been received. We use drop box software. The expert at the centre is connected with the system remotely and performs the operation by guiding the sono-therapist far apart.

- If the centre has all the equipments required to perform the task such as a computer or digital interface and equipment for communication then the total cost will include only the internet video server, camera cost, in short only the cost of communication.

\section{Tele-Medicine in DeVeloping CounTRIES like AfriCA}

The very idea of tele-health has been very grateful to the countries like Africa where good health care is not provided. Hence this helps in sharing medical issues and knowledge in the electronic form and provides access to remote expertise.

These tools are essential in places where there are no good health centres or the doctors are too far from the place and where the transportation is a big issue.

Before launching this idea on a big scale one must try this on a small scale. Some experiences in this field include:

- Video conferencing for tele-cardiology and tele-neurology in Senegal.

- Another project RAFT is going on that focuses on low cost tele-medicine. Project RAFT's goals were:

- To set up a connection between the regional and the national health care institution

- Feasibility of long distance collaboration for continuing medical education.

- Web portals to train users

- Use of low band width internet based system

The objectives of this:

1. The very main aim of this project was to motivate the doctors to go to remote places where they were actually needed and this is happened when the access of internet was provided in these remote places.

2. Medical education and updates in such places as most information publishes are not available at this location.

3. The accessibility to medical knowledge in such areas can help in creation of locally adapted medical content. 
After the implementation of this project many new countries have also joined to help in the development of this project. The Africans and European partners are satisfied with their initiative.

There might be possibility of exchange of some inadequate information. These risks have to be kept in mind while designing the tele-medicine system. Tele-medicine tools have improved the quality of medicine in remote areas. Satellite technologies used to cover the remote areas. All these are incorporated in RAFT.

\section{Tele-Medicine in Developing Countries like China}

The country of china is facing a problem regarding health issue where the farmers of the country are getting ill due to the aquaculture ie fish farming.

The diseases are commonly resulted from nutritional and environmental problems as well as infections by parasites, viruses, bacteria and fungal agents. This is leading to increase in number of patients. The fishes that are not treated on time die quickly and the farmers may not know that they are infected at the initial stage.

Therefore tele health is being used by the farmers to have a regular check up regarding the symptoms or infection.

\section{IOT IN HEALTHCARE}

Due to increasing population it has become very difficult to manage the healthcare system. Hence a new idea of a patient centric IOT healthcare system is developed.

Benefits of developing IOT e-healthcare system:

- IOTe-health provides a platform for different technologies to gathered together to work without noticing its complexity.

- Big data and analytics is also useful in our system as we require a very large database to store information of various patients.

- Personalized healthcare and treatment can be possible with help of IOT and big data analysis.

- Lifetime evaluation and monitoring of the patient.

- Easy to use by the user as is consist of only clicks and sensors.

IOTe-health care system consists of following layer:

- Device layer

- Fog layer

- Cloud layer

Device layer

- Physical Sensors:

They can be wired or wireless also that tracks patients physical wellness and digitally monitors their health. Some of them are blood pressure sensor,heart sensor, glucose sensor, hemoglobin sensor,etc.

- Virtual Sensor:

Software mobile applications and e-health services. It monitors patient's health data from the environment. This includes remote monitoring, remote consultation, nutrition etc.

\section{Fog layer}

This layer provides:

- Connectivity

- Two way exchange

- Flexible integration

- Protocol translation

- Security and data protection

- Short time data base 


\section{Cloud layer}

This layer provides the following benefits in the ehealthcare system:

- Connectivity this gives the flexibility to choose the communication method that is appropriate for the given health application.

- Data management: it integrates data from multiple sources and keeps the data safe. Hence patient health care team and anyone can access the data anytime anywhere.

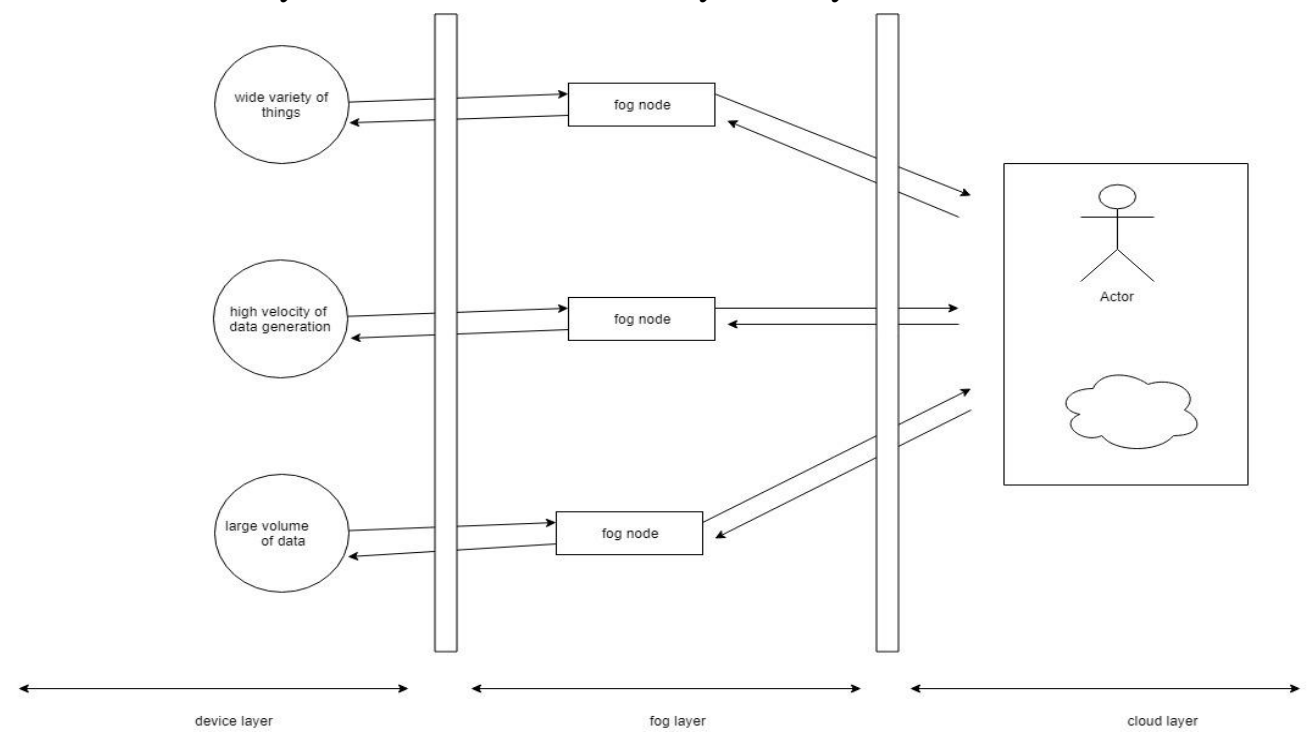

Figure3. How to read the emotional disturbance of the user

IOT is considered to be very beneficial and useful in case of e-health services

A typical IOT consist of:

1. Sensing layer

2. Networking layer

3. Service layer

4. Interface layer

Most of the work now a days is been done on the device layer as it is easy available in the market for example, smart wearable devices, fitness monitoring devices etc,

Despite of many advantages and success of IOT in field of health care we have many challenges too that are faced by IOT.

\section{CONCLUSION}

Hence, a tele-health system is used for monitoring individuals suffering from various

diseases. This system uses technologies such as IOT (Internet of Things) \& RFID (Radio Frequency Identification). Dedicated

communication networks are required for the remote monitoring medical applications. This enables the home care tele-assistance system for senior citizens. Its aim is to make it easier for the users keep a track of their health anytime anywhere in any manner. This has helped in reducing the cost, formalities, waiting, more actions at low price as the hospital is being shifted to home and there is no need to travel to the hospital. Main target of such idea is the elderly people and people suffering from chronic disease.

The barriers in this are:

- Elderly people are new to technology

- Legal shortage 
This system provides:

- Better and easier lifestyle

- Healthcare management

- Responsibility towards health

- Medical and socio assessment

\section{REFERENCES}

[1] Knight H. Sound concept: medical spin-off from ISS technology. Engineer. 2016; Accessed 16 March 2016

[2] S.-M.S.S.-W. Kim, J.-W. Jeon, Food Contamination Monitoring via Internet of Things." Conference on, IEEE. 2015, pp. 609-613.

[3] M. Sarrafzadeh, F. Dabiri, H. Noshadi, Fast behavior and abnormality detection, 2016. US Patent $20,160,148,103$

[4] E. Smets, P. Casale, U. Grossekathoefer, B. Lamichhane, W. De Raedt, K. Bogaerts, I. Van Diest, C. Van Hoof, Comparison of machine learning techniques for psychophysiological stress detection, in: Mindcare 2015 - 5th EAI International Symposium on Pervasive Computing Paradigms for Mental Health, 2015

[5] Allen, A., Roman, L., et al., 1996. Home health visits usinga cable television network: user satisfaction. Journal of Telemedicine and Telecare 2, 92-94.

[6] Johnston, B., Wheeler, L., Deuser, J., Sousa, K.H., 2000. Outcomes of the Kaiser permanent tele-home health research project. Archives of Family Medicine 9, 40-45. Perednia DA, Allen A. Telemedicine technology and clinical applications. J Am Med Assoc 1995;274(6):461-2.

[7] Ganapathy K. Telemedicine and neurosciences in developing countries. SurgNeurol 2002;58:388-94.

[8] Graham LE, Zimmerman M, Vassallo DJ, Patterson V, Swinfen P, Swinfen R, et al. Telemedicine- the way ahead for medicine in the developing world. Trop Doctor 2003;33:36-8.

[9] Sarbadhikari SN. The state of medical informatics in India: a roadmap for optimal organization. J Med Syst 2005;29(2):125-41.

[10] Wright D. Telemedicine and developing countries. A report of study group 2 of the ITU Development Sector. J Telemed Telecare 1998;4(Suppl. 2): 1-85

[11] Meystre S, M"uller H. Open source software in the biomedical domain: electronic health records and other useful applications. Swiss Med Inform 2005;55:3-15.

[12] Brauchli K, Mohony DO, Banach L, Oberholzer M. iPath - a telemedicine platform to support health providers in low resource settings. Stud Health Technol Inform; 2005.

[13] La T'el'emedecinerem'ede 'al'isolement, Africa International, 394, janvier 2006.

[14] Kienzle M, Curry D, Franken EA, Galvin J, Hoffmam E, Holtum E, et al. University of Iowa. Iowa's National Laboratory for the study of rural telemedicine:

[15] Dansky KH, Bowles KH, \&Palmer L: How tele- homecare affects patients. Caring

[16] Chowdhury, B. and Khosla, R., 'RFID-based Hospital Real-time Elderly Management System'

[17] Dragomir R, Pucoci S, Tache M Some Aspects Regarding Elder Patient Acceptance over an ITC Mobile Platform, Adjunct Proceeding of designing Ambient Interactions for Older Users, European Conference of Ambient Intelligence.

Citation: Pravin Wararkar et al.(2020)" Tele-Health: Integrated Remote Disease Diagnosis and Treatment Platform, International Journal of Innovative Research in Electronics and Communications (IJIREC), 7(2), pp.2026, DOI: http://dx.doi.org/10.20431/2349-4050.0702004

Copyright: ( 2020 Pravin Wararkar. This is an open-access article distributed under the terms of the Creative Commons Attribution License, which permits unrestricted use, distribution, and reproduction in any medium, provided the original author and source are credited 